\title{
ESTUDO DOS EFEITOS DA TEMPERATURA, TEMPO DE REAÇÃO E ESTEQUIMETRIA NA SÍNTESE DE NANOBASTÕES DE ZnO PARA APLICAÇÃO EM FOTOCATÁLISE
}

\author{
M. BAGNARA ${ }^{1}$ e M. A. LANSARIN ${ }^{1}$ \\ ${ }^{1}$ Universidade Federal do Rio Grande do Sul, Departamento de Engenharia Química \\ E-mail para contato: monica@enq.ufrgs.br
}

\begin{abstract}
RESUMO - Neste trabalho foram preparadas microestruturas de $\mathrm{ZnO}$, imobilizadas sobre placas de zinco, visando sua aplicação na fotocatálise. A síntese foi realizada através de tratamento hidrotérmico e rodamina B foi escolhida como molécula teste para avaliação da atividade catalítica dos materiais. Um planejamento de experimentos composto central circunscrito foi utilizado para estudar as variáveis de síntese do tratamento hidrotérmico (razão molar $\mathrm{NaOH} / \mathrm{Zn}\left(\mathrm{NO}_{3}\right)_{2}$, tempo, temperatura e razão molar $\mathrm{Zn}\left(\mathrm{NO}_{3}\right)_{2}$ /frutose). Observou-se que a quantidade de $\mathrm{NaOH}$ presente durante a síntese é a variável mais importante, apresentando fatores lineares e quadráticos significativos. A melhor condição do planejamento de experimentos estudado possibilitou a remoção de $80 \%$ de corante em uma hora de exposição à radiação UV. As imagens de MEV mostram a obtenção de diferentes estruturas e a análise dos difratogramas indicam a presença de fases cristalinas.
\end{abstract}

\section{INTRODUÇÃO}

A fotocatálise é um processo oxidativo avançado muito promissor para aplicação na degradação de contaminantes orgânicos, uma vez que, em condições adequadas de operação, resulta na produção de $\mathrm{CO}_{2}, \mathrm{H}_{2} \mathrm{O}$ e ácidos alifáticos de baixo peso molecular (Behnajady et al., 2007). Além disso, pode ser aplicada na redução de contaminantes metálicos (Chakrabarti et al., 2009) e na inativação de bactérias, tanto em fase gasosa (Pal et al., 2008), quanto líquida (Byrne et al., 2011).

Entre os catalisadores usados em fotocatálise estão $\mathrm{TiO}_{2}, \mathrm{ZnO}, \mathrm{Fe}_{2} \mathrm{O}_{3}, \mathrm{CdS}, \mathrm{ZnS}$ e GaP, sendo que o dióxido de titânio é o mais estudado e mais ativo sob radiação entre $300 \mathrm{~nm}$ e $390 \mathrm{~nm}$, além de ser estável em vários ciclos catalíticos (Chong et al., 2010). Entretanto, o óxido de zinco possui características peculiares que o tornam interessante em muitas pesquisas. A maior vantagem do $\mathrm{ZnO}$ em relação ao $\mathrm{TiO}_{2}$ é a capacidade de absorver uma fração maior do espectro UV (Behnajady et al., 2007).

Quando o catalisador é usado em suspensão, uma etapa posterior para separação do sólido se faz necessária, o que onera o processo, dificultando uma possível aplicação em maior escala. Portanto, a obtenção de catalisador imobilizado, pelo seu potencial de reduzir custos de operação, é de grande interesse e vem sendo investigada. Entre as técnicas encontradas na literatura estão: crescimento na 


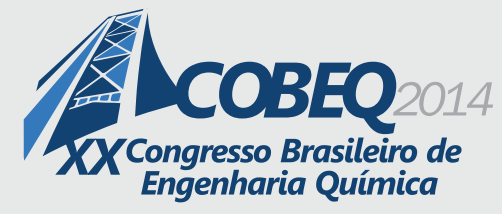

presença de templetes (Xie et al., 2012), fotolitografia (Szabó et al., 2013), eletrodeposição (Lu et al., 2014), deposição química de vapor (Mohanta et al., 2014), evaporação térmica (Li et al., 2013; Jumidali e Hashim, 2012), oxidação de lâminas de zinco em fase gasosa e aquosa (Tan et al., 2011) e rotas hidrotérmicas (Zhao et al., 2014; Ma et al., 2011).

A rota hidrotérmica apresenta a vantagem de não necessitar nenhuma espécie de camada semente no substrato, ser conduzida a temperaturas relativamente baixas e com reagentes de fácil acesso. Além disso, possibilitam a obtenção de estruturas ordenadas de $\mathrm{ZnO}$ imobilizadas sobre diferentes substratos, dentre eles lâminas de alumínio (Kaneva et al., 2012), lâminas de cobre (Ma et al., 2011), lâminas de zinco (Hou et al., 2007), vidro (Kenanakis et al., 2009), FTO (Wang et al., 2010), ITO (Guo et al., 2007) e quartzo (Xie et al., 2011).

O principal objetivo deste trabalho foi estudar os efeitos das condições de síntese de $\mathrm{ZnO}$ imobilizado em folhas de zinco metálico, através do tratamento hidrotérmico, na atividade fotocatalítica. A atividade fotocatalítica das amostras de $\mathrm{ZnO}$ sintetizadas foi avaliada através da degradação fotocatalítica de rodamina B sob radiação UV.

\section{PARTE EXPERIMENTAL}

\subsection{Materiais}

Todos os reagentes foram utilizados como adquiridos. Nitrato de zinco $\mathrm{Zn}\left(\mathrm{NO}_{3}\right)_{2} \cdot 6 \mathrm{H}_{2} \mathrm{O}$ e Dfrutose foram adquiridas da Synth com $98 \%$ de pureza, $\mathrm{NaOH}$ da Nuclear, com $97 \%$ de pureza. Etanol, 2-propanol e acetona usados foram da Dinâmica, e o corante rodamina B da Próton-Química.

\subsection{Síntese de $\mathrm{ZnO}$ imobilizado}

As lâminas de zinco $(2,5 \mathrm{~cm}$ X 2,5 cm) usadas como substrato foram inicialmente limpadas em banho de ultrassom com uma sucessão de solventes (acetona, 2-isopropanol e etanol). Para a síntese de $\mathrm{ZnO}$ imobilizado, inicialmente preparou-se uma suspensão com $\mathrm{Zn}\left(\mathrm{NO}_{3}\right)_{2} \cdot 6 \mathrm{H}_{2} \mathrm{O} \quad 0,5$ mol. L $\mathrm{L}^{-1}$ e $\mathrm{NaOH}$ que foi mantida em agitação por cinco minutos. Em seguida, adicionou-se D-frutose à suspensão e mantida a agitação por mais trinta minutos. A suspensão foi então transferida para autoclaves de aço inoxidável com revestimento de tetrafluoretileno, nas quais o substrato é acomodado inclinado na base. As amostras foram submetidas ao tratamento hidrotérmico por diferentes períodos de tempo (1 a 48 horas), e temperatura $\left(55\right.$ a $\left.115^{\circ} \mathrm{C}\right)$. Ao término da reação, as lâminas com o semicondutor foram lavadas suavemente com água deionizada para remover o excesso de sólidos e secadas a $60{ }^{\circ} \mathrm{C}$ durante a noite.

\subsection{Planejamento de Experimentos}

Um planejamento de experimentos de composto central circunscrito foi desenvolvido de modo a melhor compreender o efeitos das variáveis de síntese, razão molar $\mathrm{NaOH} / \mathrm{Zn}\left(\mathrm{NO}_{3}\right)_{2}$, tempo (h), temperatura $\left({ }^{\circ} \mathrm{C}\right)$ e razão molar $\mathrm{Zn}\left(\mathrm{NO}_{3}\right)_{2} /$ frutose, na atividade fotocatalítica dos materiais. A variável de resposta escolhida foi a porcentagem de degradação fotocatalítica de rodamina B. Este modelo 


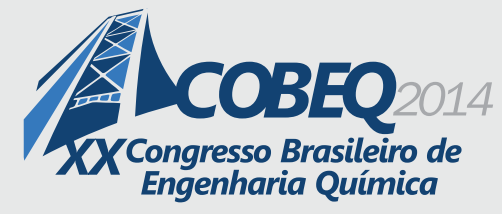

consiste de um planejamento factorial com $2^{\mathrm{n}}(\mathrm{n}=4)$ experimentos, 8 pontos estrela e 3 pontos centrais, resultando em um total de 27 experimentos. Os dados foram analisados através do software Statistica 8 , com um intervalo de confiança de $90 \%$, que fornece o polinômio associado ao modelo.

\subsection{Determinação da Atividade Fotocatalítica}

A atividade fotocatalítica das amostras de $\mathrm{ZnO}$ sintetizadas foi avaliada através da decomposição de rodamina B sob radiação UV. Inicialmente, as lâminas com $\mathrm{ZnO}$ imobilizado foram imersas em $40 \mathrm{~mL}$ de uma solução de rodamina B $10 \mathrm{mg} . \mathrm{L}^{-1}$ e o sistema foi mantido no escuro por sessenta minutos para estabelecimento do equilíbrio de adsorção. Em seguida, o sistema foi irradiado com uma lâmpada de vapor de mercúrio de $125 \mathrm{~W}$ por sessenta minutos. A irradiação da lâmpada foi medida e ajustada em aproximadamente $3,5 \mathrm{~mW} . \mathrm{cm}^{-2}$, no comprimento de onda de $365 \mathrm{~nm}$, ao início de cada reação, utilizando para isto um radiômetro (Cole-Parmer Instrument, Series 9811 Radiometer). A temperatura do sistema foi mantida constante a $30^{\circ} \mathrm{C}$ através de um banho termostático. A absorbância máxima do corante foi medida em espectrofotômetro de feixe simples (Pró-Análise, UV-1600) a $\lambda=553 \mathrm{~nm}$.

\subsection{Caracterização}

A estrutura cristalina das amostras foi analisada através de difração de raios $\mathrm{X}$ (DRX), utilizando-se um difratômetro Bruker, modelo D2 Phaser, com radiação CuKa. As amostras foram escaneadas na faixa de $2 \theta$ de $20^{\circ}$ a $70^{\circ}$, com uma tensão de aceleração e corrente aplicada de $30 \mathrm{kV}$ e $10 \mathrm{~mA}$, respectivamente. Uma vez que a estrutura cristalina independe do substrato, o material sedimentado no fundo das autoclaves foi utilizado para DRX.

A morfologia foi estudada através de microscopia eletrônica de varredura (MEV). As análises de MEV foram realizadas no Centro de Microscopia Eletrônica da Universidade Federal do Rio Grande do Sul. O microscópio utilizado foi JOEL JSM 5800 e as amostras foram analisadas conforme sintetizadas, sem necessidade de remoção do filme.

\section{RESULTADOS}

\subsection{Planejamento de Experimentos}

A Tabela 1 apresenta os experimentos realizados e a degradação alcançada de rodamina B. As variáveis estudadas foram concentração de $\mathrm{NaOH}(\alpha)$, tempo de reação $(\beta)$, temperatura de reação $(\gamma)$ e concentração de D-frutose $(\omega)$. Observa-se que os maiores percentuais de degradação (80 e $79 \%$ ) foram alcançados quando se empregou os catalisadores preparados usando-se uma concentração alta de frutose, e os níveis superiores de temperatura e concentração de $\mathrm{NaOH}$ (experimentos 6 e 8). Salienta-se que estes dois materiais permitiram alcançar aproximadamente a mesma degradação embora tenham sido sintetizados usando-se os níveis superior e inferior de tempo de síntese. Na outra ponta, na menor faixa de degradação (13\%), está a amostra obtida usando-se o catalisador preparado com condições de tempo, temperatura e concentração de frutose idênticas às do experimento 6 , mas estando a concentração de $\mathrm{NaOH}$ no seu nível mais baixo (experimento 5). 


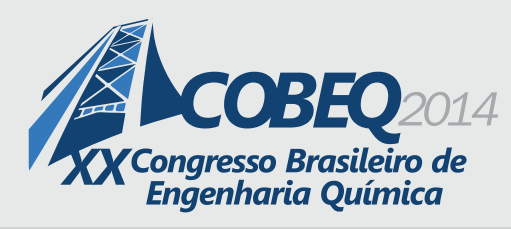

19 a 22 de outubro de 2014

Florianópolis/SC

Tabela 1 - Códigos das variáveis utilizadas no planejamento de experimentos e a resposta obtida

\begin{tabular}{|c|c|c|c|c|c|}
\hline Experimento & $\alpha$ & $\beta$ & $\gamma$ & $\omega$ & \% Degrad. \\
\hline 1 & $2(-1)$ & $12 \mathrm{~h}(-1)$ & $75^{\circ} \mathrm{C}(-1)$ & $4(-1)$ & $16 \%$ \\
\hline 2 & $6(+1)$ & $12 \mathrm{~h}(-1)$ & $75^{\circ} \mathrm{C}(-1)$ & $4(-1)$ & $54 \%$ \\
\hline 3 & $2(-1)$ & $36 \mathrm{~h}(+1)$ & $75^{\circ} \mathrm{C}(-1)$ & $4(-1)$ & $20 \%$ \\
\hline 4 & $6(+1)$ & $36 \mathrm{~h}(+1)$ & $75^{\circ} \mathrm{C}(-1)$ & $4(-1)$ & $60 \%$ \\
\hline 5 & $2(-1)$ & $12 \mathrm{~h}(-1)$ & $115^{\circ} \mathrm{C}(+1)$ & $4(-1)$ & $13 \%$ \\
\hline 6 & $6(+1)$ & $12 \mathrm{~h}(-1)$ & $115^{\circ} \mathrm{C}(+1)$ & $4(-1)$ & $80 \%$ \\
\hline 7 & $2(-1)$ & $36 \mathrm{~h}(+1)$ & $115^{\circ} \mathrm{C}(+1)$ & $4(-1)$ & $32 \%$ \\
\hline 8 & $6(+1)$ & $36 \mathrm{~h}(+1)$ & $115^{\circ} \mathrm{C}(+1)$ & $4(-1)$ & $79 \%$ \\
\hline 9 & $2(-1)$ & $12 \mathrm{~h}(-1)$ & $75^{\circ} \mathrm{C}(-1)$ & $14(+1)$ & $25 \%$ \\
\hline 10 & $6(+1)$ & $12 \mathrm{~h}(-1)$ & $75^{\circ} \mathrm{C}(-1)$ & $14(+1)$ & $53 \%$ \\
\hline 11 & $2(-1)$ & $36 \mathrm{~h}(+1)$ & $75^{\circ} \mathrm{C}(-1)$ & $14(+1)$ & $8 \%$ \\
\hline 12 & $6(+1)$ & $36 \mathrm{~h}(+1)$ & $75^{\circ} \mathrm{C}(-1)$ & $14(+1)$ & $61 \%$ \\
\hline 13 & $2(-1)$ & $12 \mathrm{~h}(-1)$ & $115^{\circ} \mathrm{C}(+1)$ & $14(+1)$ & $11 \%$ \\
\hline 14 & $6(+1)$ & $12 \mathrm{~h}(-1)$ & $115^{\circ} \mathrm{C}(+1)$ & $14(+1)$ & $49 \%$ \\
\hline 15 & $2(-1)$ & $36 \mathrm{~h}(+1)$ & $115^{\circ} \mathrm{C}(+1)$ & $14(+1)$ & $18 \%$ \\
\hline 16 & $6(+1)$ & $36 \mathrm{~h}(+1)$ & $115^{\circ} \mathrm{C}(+1)$ & $14(+1)$ & $58 \%$ \\
\hline 17 & $0(-2)$ & $24 \mathrm{~h}(0)$ & $95^{\circ} \mathrm{C}(0)$ & $9(0)$ & $20 \%$ \\
\hline 18 & $8(+2)$ & $24 \mathrm{~h}(0)$ & $95^{\circ} \mathrm{C}(0)$ & $9(0)$ & $57 \%$ \\
\hline 19 & $4(0)$ & Oh $(-2)$ & $95^{\circ} \mathrm{C}(0)$ & $9(0)$ & $30 \%$ \\
\hline 20 & $4(0)$ & $48 \mathrm{~h}(+2)$ & $95^{\circ} \mathrm{C}(0)$ & $9(0)$ & $68 \%$ \\
\hline 21 & $4(0)$ & $24 \mathrm{~h}(0)$ & $55^{\circ} \mathrm{C}(-2)$ & $9(0)$ & $35 \%$ \\
\hline 22 & $4(0)$ & $24 \mathrm{~h}(0)$ & $135^{\circ} \mathrm{C}(+2)$ & $9(0)$ & $59 \%$ \\
\hline 23 & $4(0)$ & $24 \mathrm{~h}(0)$ & $95^{\circ} \mathrm{C}(0)$ & $0(-2)$ & $28 \%$ \\
\hline 24 & $4(0)$ & $24 \mathrm{~h}(0)$ & $95^{\circ} \mathrm{C}(0)$ & $18(+2)$ & $56 \%$ \\
\hline 25 & $4(0)$ & $24 \mathrm{~h}(0)$ & $95^{\circ} \mathrm{C}(0)$ & $9(0)$ & $62 \%$ \\
\hline 26 & $4(0)$ & $24 \mathrm{~h}(0)$ & $95^{\circ} \mathrm{C}(0)$ & $9(0)$ & $68 \%$ \\
\hline 27 & $4(0)$ & $24 \mathrm{~h}(0)$ & $95^{\circ} \mathrm{C}(0)$ & $9(0)$ & $68 \%$ \\
\hline
\end{tabular}

A análise de dados feita através do software Statistica, utilizando a ferramenta ANOVA, forneceu um coeficiente de correlação relativamente baixo, em torno de 0,8 , indicando que o modelo não é preditivo em relação aos dados experimentais. Mesmo assim, as superfícies de resposta elucidam o comportamento das variáveis de síntese. Como se pode ver na Figura 1, as maiores reduções são obtidas quando se usa as maiores quantidades de $\mathrm{NaOH}$ na síntese dos materiais associadas à quantidades intermediárias de frutose. Este comportamento é corroborado pelos experimentos 6 e 8. Além disso, temperaturas entre 100 e $120^{\circ} \mathrm{C}$, associadas a tempos de síntese entre 10 e 40h também levam a reduções elevadas, como pode ser visto na Figura 1b e nos experimentos 6 e 8 .

Por outro lado, usando-se as condições de tempo, temperatura e concentração de frutose do 
experimento 6 , mas baixa concentração de $\mathrm{NaOH}$, observa-se a menor redução do corante: situação correspondente ao experimento 5 e a zona alaranjada da Figura 1a. Comparando-se os experimentos 5 e 13, conclui-se que a variável mais importante para a síntese é a concentração de $\mathrm{NaOH}$.

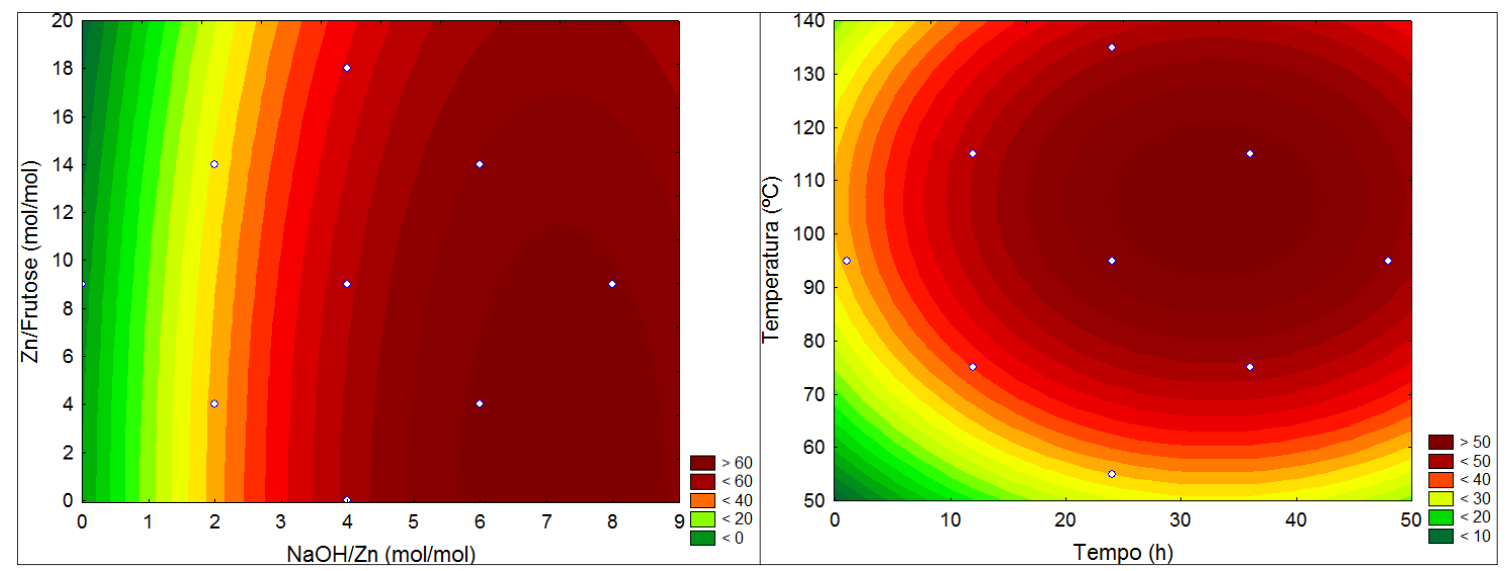

Figura 1 - Gráficos de contorno para o planejamento de experimentos composto central circunscrito.

\subsection{Caracterização}

A cristalografia dos depósitos sólidos presentes no fundo das autoclaves foi analisada por difração de raios $\mathrm{X}$, e os difratogramas de algumas amostras do planejamento de experimentos são apresentados na Figura 2. As amostras estudadas apresentam estrutura cristalina, e todos os picos presentes nos difratogramas podem ser atribuídos à estrutura hexagonal wurtzita. Observa-se que os picos mais intensos referem-se à amostra do ponto 6, amostra esta que também apresentou a maior degradação fotocatalítica de rodamina $B$.

A morfologia do material depositado sobre as placas de zinco foi analisada por MEV e a Figura 3 apresenta algumas micrografias obtidas neste estudo, todas com uma ampliação de 10.000 vezes. Pode-se constatar nestas imagens a formação de diferentes estruturas. Para a amostra 13 do planejamento de experimentos, associada a mais baixa degradação de corante e apresentada na Figura 18b, não se observa um padrão na formação do sólido. Já na imagem correspondente ao ponto 18, há a formação de uma estrutura em forma de flor, composta de microbastões hexagonais de $\mathrm{ZnO}$. Entretanto, as amostras referentes aos pontos 6 e 25 (18a e 18d, respectivamente), apresentaram uma estrutura pouco regular, mas uma elevada atividade fotocatalítica, com 80 e $69 \%$ de degradação de rodamina em uma hora de reação. 


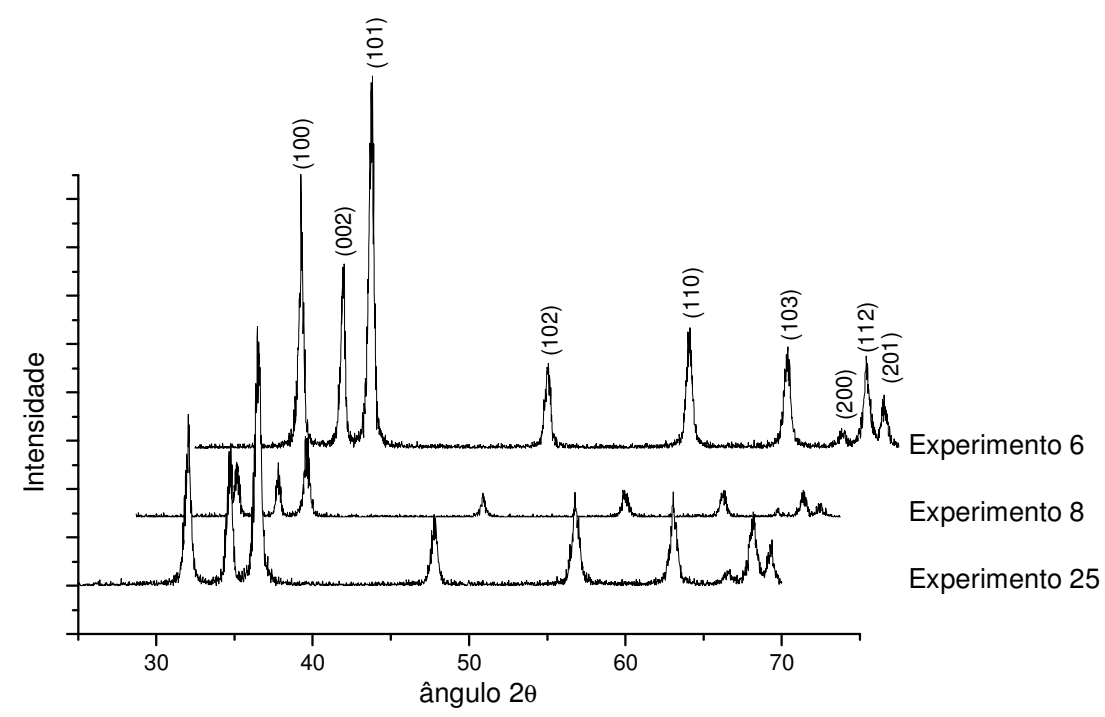

Figura 2 - Difratogramas dos resíduos das amostras de diferentes pontos do planejamento de experimentos.

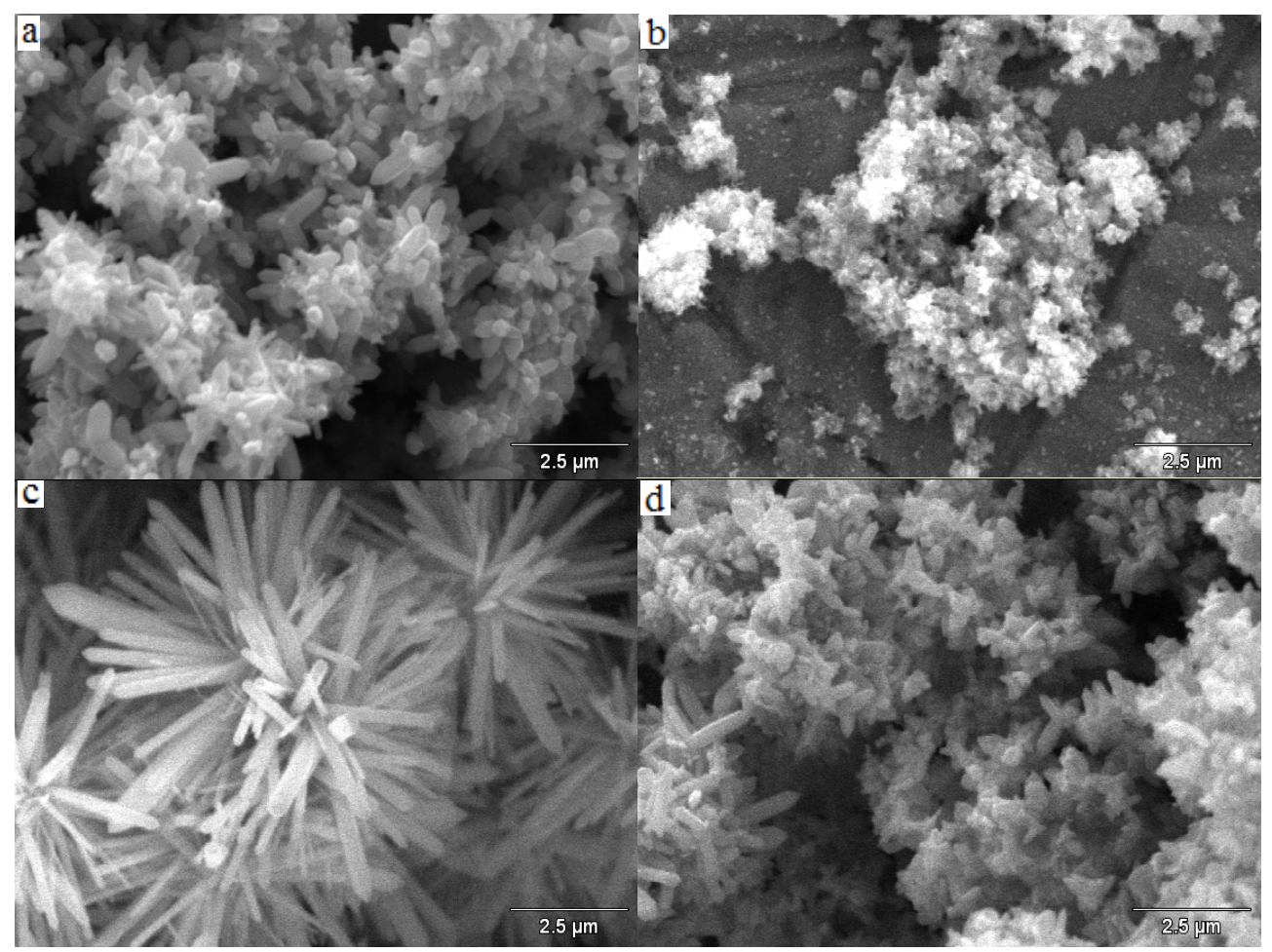

Figura 4 - MEV dos experimentos 6 (a), 13 (b), 18 (c) e 25 (d). 


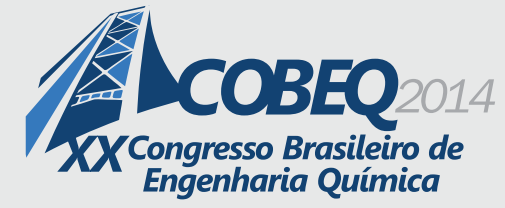

Devido à anisotropia estrutural e a polaridade eletrônica de superfície do $\mathrm{ZnO}$, a maior taxa de crescimento é ao longo do plano [ $\left[\begin{array}{llll}0 & 0 & 0 & 1\end{array}\right]$, e deste modo obtém-se predominantemente estruturas na forma de bastões hexagonais alongados na direção do eixo $c$, apresentando uma polaridade $\mathrm{O}^{2-}$ ou $\mathrm{Zn}^{2+}$ (Thao et al, 2010; Ahsanulhaq et al., 2009). Os planos [ $\left[\begin{array}{llll}1 & 0 & -1 & 0\end{array}\right]$ são compostos de quantidades equivalentes de íons $\mathrm{O}^{2-}$ e $\mathrm{Zn}^{2+}$, razão pela qual estes planos nãopolares têm menor energia de superfície e menor capacidade de absorver espécies como oxigênio. A maior capacidade de degradação de corante referente às amostras 6 e $25(80 \%$ e 68\%, respectivamente) pode ser associada aos bastões mais largos, uma vez que há uma superfície maior com íons $\mathrm{Zn}^{2+}$ expostos, possibilitando uma maior adsorção de espécies como $\mathrm{O}^{-}, \mathrm{O}^{2-}, \mathrm{O}_{2}$ e $\mathrm{HO}^{-}$, aumentando a atividade fotocatalítica.

\section{CONCLUSÕES}

O presente trabalho relata o estudo das condições de síntese de microestruturas de $\mathrm{ZnO}$ imobilizadas sobre placas de zinco para aplicação na degradação fotocatalítica de corante orgânico, através de um planejamento de experimentos de composto central circunscrito. Através dos gráficos de contorno foi possível verificar uma região de maior resposta, sendo que a variável mais importante na síntese é a razão molar $\mathrm{NaOH} / \mathrm{Zn}$. A análise do resíduo de fundo das autoclaves mostra a presença de estruturas cristalinas, correspondentes à estrutura hexagonal wurtiza. As micrografias obtidas indicam que a baixa concentração de $\mathrm{NaOH}$ dificulta a formação de microbastões hexagonais, como observado na Figura 18b. A melhor atividade fotocatalítica foi obtida com a amostra 6, possibilitando uma remoção de $80 \%$ de corante em uma hora de reação.

\section{AGRADECIMENTOS}

As autoras agradecem a CAPES pelo apoio financeiro para a realização da presente pesquisa.

\section{REFERÊNCIAS}

BEHNAJADY, M.A.; MODIRSHAHLA, N.; DANESHVAR, N.; RABBANI, M. Photocatalytic degradation of C.I. Acid Red 27 by immobilized $\mathrm{ZnO}$ on glass plates in continuous-mode. J. Hazardous Materials, v. 140, p. 257-263, 2007.

BYRNE J. A.; FERNANDEZ-IBAÑEZ, P. A.; DUNLOP, P. S.M.; ALROUSAN, D. M. A.; HAMILTON, J. W. J. Photocatalytic Enhancement for Solar Disinfection of Water: A Review. International Journal of Photoenergy, 2011. doi:10.1155/2011/798051.

CHAKRABARTI, S.; CHAUDHURI, B.; BHATTACHARJEE, S.; RAY. A. K.; DUTTA, B. K. Photo-Reduction of Hexavalent Chromium in Aqueous Solution in the Presence of Zinc Oxide as Semiconductor Catalyst. Chem. Eng. J., v. 153, p. 86-93, 2009.

CHONG, M. N.; JIN, B.; CHOW, C. W. K.; SAINT, C. Recent developments in photocatalytic water treatment technology: A Review. Water Research, v. 44, p. 2997 - 3027, 2010.

JUMIDALI, M. M.; HASHIM, Md. R. Modified Thermal Evaporation Process Using GeO2 for Growing ZnO Structures. Superlattices and Microstructures, v. 52, p. 33-40, 2012. 
KANEVA, N.; STAMBOLOVA, I.; BLASKOV, V.; DIMITRIEV, Y.; BOJINOVA, A.; DUSHKIN, C. A comparative study on the photocatalytic efficiency of $\mathrm{ZnO}$ thin films prepared by spray pyrolysis and sol-gel method. Surface \& Coatings Technology, v. 207, p. 5-10, 2010.

KENANAKIS, G.; VERNARDOU, D.; KOUDOUMAS, E.; KATSARAKIS, N. Growthof c-axis oriented $\mathrm{ZnO}$ nanowires from aqueous solution: The decisive role of a seed layer for controlling the wires' diameter. Journal of Crystal Growth, v. 311, p. 4799-4804, 2009.

LI, Q.; CHEN, Y.; LUO, L.; WANG, L.; YU, Y.; ZHAI, L. Photoluminescence and Wetting Behavior of $\mathrm{ZnO}$ Nanoparticles/Nanorods Array Synthesized by Thermal Evaporation. Journal of Alloys and Compounds, v. 560, p. 156-160, 2013.

LU, H.; ZHENG, F.; GUO, M.; ZHANG, M. One-Step Electrodeposition of Single-Crystal ZnO Nanotube Arrays and Their Optical Properties. Journal of Alloys and Compounds, v. 588, p. 217-221, 2014.

MA, S.; LI, R.; LV, C.; XU, W; GOU, X.. Facile Synthesis of ZnO Nanorod Arrays and Hierarchical Nanostructures for Photocatalysis and Gas Sensor Applications. Journal of Hazardous Materials, v. 192, p. 730-740, 2011.

MOHANTA, A.; SIMMONS Jr., J. G.; EVERITT, H. O.; SHEN, G.; KIM, S. M.; KUNG, P. Effect of Pressure and Al Doping on Structural and Optical Properties of ZnO Nanowires Synthesized by Chemical Vapor Deposition. Journal of Luminescence, v. 146, p. 470-474, 2014.

PAL, A.; PEHKONEN, S. O.; YU, L. E.; RAY, M. B. Photocatalytic Inactivation of Airborne Bacteria in a Continuous-Flow Reactor. Industrial \& Engineering Chemistry Research, v. 47, p. 7580-7585, 2008.

SZABÓ, Z; VOLK, J.; FÜLÖP, E.; BARSONY, I. Regular ZnO Nanopillar Arrays by Nanosphere Photolithography. Photonics and Nanoestructures - Fundamentals and Applications, v. 11, p. 1-7, 2013.

TAN, W.K.; RAZAK, K. Abdul; IBRAHIM, K.; LOCKMAN, Zainovia. Oxidation of Etched Zn Foil for the Formation of ZnO Nanostructure. Journal of Alloys and Compounds, v. 509, p. 6806-6811, 2011.

WANG, S.L.; JIA, X.; JIANG, P.; FANG, H.; TANG, W.H. Large-scale preparation of chestnutlike $\mathrm{ZnO}$ and $\mathrm{Zn}-\mathrm{ZnO}$ hollow nanostructures by chemical vapor deposition. Journal of Alloys and Compounds, v. 502,p. 118-122, 2010.

XIE, Yu; CAI, Bing; YU, Donggi; SHAN, Whenjuan; ZHANG, Wen-Hua. Template-Guided Growth of Well-Aligned ZnO Nanocone Arrays on FTO Substrate. Journal of Crystal Growth, v. 346, p. 64-68, 2012.

ZHAO, X.; LOU, F.; LI, M.; LOU, X.; LI, Z.; ZHOU, J. Sol-Gel-Based Hydrothermal Method for the Synthesis of 3D Flower-Like ZnO Microstructures Composed of Nanosheets for Photocatalytic Applications. Ceramics International, v. 40, p. 5507-5514, 2014. 